$\xi=1$ 圆

\title{
Informational Description of Systemic Crises
}

\author{
Yudenkov A.V. ${ }^{1^{*}}$, Terentyev S.E. ${ }^{2}$, Kovaleva A.E. ${ }^{3}$ \\ ${ }^{l}$ Professor, Doctor of Physical and Mathematical Sciences (Advanced Doctor), \\ Smolensk State Agricultural Academy. \\ ${ }^{2}$ Associate Professor, Candidate of Agricultural Sciences (Ph.D.), Smolensk State Agricultural Academy. \\ ${ }^{3}$ Candidate of Economic Sciences (Ph.D.), Smolensk State Agricultural Academy. \\ *Corresponding authorE-mail: ecolog_777@mail.ru
}

\begin{abstract}
The paper studies the possibility of determining and classifying the crisis as a complex system by a remote observer on the basis of subjective information. Description and analysis of complex systems is a fundamentally unsolvable problem. However, there may be a partial solution to the problem through the use of multilevel modeling. Therefore, the development of new fairly common methods for modeling complex systems is an urgent task. The aim of the work is to develop fairly common methods of modeling complex systems in crisis. For this purpose, the evolution of the system is considered at three levels: micro level, meso level and macro level. At the micro level such concepts as unit of information, growth of information are considered. At the macro level, two models describing system crises are proposed. The simulation is based on stochastic differential equations and the theory of phase transitions. At the micro level, the process of transition from one stable state to another is studied. It is assumed that the remote macroscopic observer receives information about the evolution of the system. The new results include the following. A new interpretation of information from the quantum-statistical point of view. Unlike Shannon's information in this paper, the information is associated with the phase space of the system. This makes it possible to apply basic physical and mathematical methods to the study of the evolution of different nature of systems. An analogue of the second principle of thermodynamics at the micro level-the principle of maximum information is obtained. The obtained results allowed justifying the use of Langevin equations for crisis modeling, as well as to obtain an analogy between the types of crises and phase transitions. The paper considers illustrating examples of complex systems in the process of transition from one stable state to another.
\end{abstract}

Keywords: analysis, Brownian motion, entropy, system crisis, Heisenberg uncertainty, phase space, phase transitions, Langevin equation.

\section{Introduction}

Modeling of complex systems is a task that is far from being solved at the moment. The paper presents one of the possible options for describing a complex system at the time of crisis.

It is known that the functioning of a complex system is fundamentally impossible to describe with one universal model. Therefore, it is advisable to apply several mutually related models. This allows studying the processes taking place in the system from different sides, without losing the generality of the description.

One of the effective methods of modeling is the division of a complex system into levels (macro level, micro level, meso level). At the macro level, the system is studied as a whole. At the micro level, the individual elements of the system are considered. The meso level describes the dynamics of the processes.

In work, the crisis is understood as the process of transition of a complex system from one stable state to another. At the same time, the main characteristic of the crisis is the change in the information recorded by a remote macroscopic observer. This approach is the most common and will be effective for systems of different nature.

The theoretical justification of the proposed model is given at the micro level. The connection of information and entropy is shown, the method of substantiation of the principle of maximum information with the use of discrete phase space is proposed.
The results are used to build a macro-level model and describe the dynamics of the crisis.

\section{Purpose and objectives of the study}

Substantiate and build a general multi-level model of the crisis as a complex system based on the analysis of changes in information. The purpose of the study involves the following objectives.

1. Definition of the unit of information using discrete phase space.

2. Determination of the basic laws of increasing information.

3. Classification of crises and description of their course.

\section{Methods and materials}

The main methods of research were: general theory of systems, statistical physics, theory of stochastic differential equations, and theory of phase transitions of LGD.

\section{The results and their discussion}

Are divided into several interrelated topics in the paper. 


\subsection{Source of uncertainty}

Let us recall that the main function of any open system from the point of view of an outside observer is to convert the input signal to the output (Fig. 1).

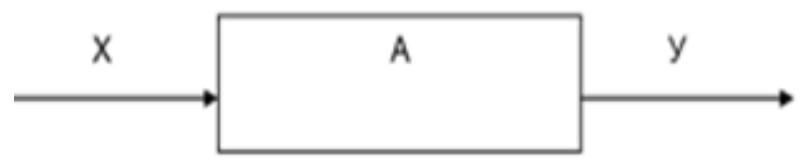

Fig. 1: General operating model of the system

Here $\mathrm{X}$ denotes the space of input signals, $\mathrm{Y}$ - the space of output signals, and $\mathrm{A}$ - the system operator. The mathematical model of the system can be presented in the following form ([10] p.26):

$$
y(t)=A x(t)
$$

How can the system operator responsible for its functioning be identified? The classical theory of systems answers this question as follows. The pulse signal $\delta(t)$ function must be input. Then the following ratio is true:

$$
y(t)=A x(t)=A \int_{-\infty}^{+\infty} x(\tau) \delta(\tau-t) d \tau=\int_{-\infty}^{+\infty} x(\tau) A[\delta(\tau-t)] d \tau
$$

It is almost impossible to influence the system with an instantaneous signal having infinitely large energy. The real signal has a certain energy $\Delta \mathrm{E}$ and lasts for a period $\Delta \mathrm{t}$. This leads to the fact that in the process of obtaining information by an external observer, the system changes randomly, while changing the output signal. Therefore, even the deterministic system from the point of view of an external observer is stochastic.

To assess the uncertainty, we use the concept of action (phase volume)

$$
S=E t
$$

Or

$$
S=p_{r}
$$

Using the Heisenberg relation ([7]p.70, [8]P. 41), we obtain the minimum size of the action (the minimum phase cell):

$$
S_{0}=h^{n}
$$

Here $\mathrm{h}$ - Planck constant, $\mathrm{n}-$ the number of degrees of freedom of the system. To go to the concept of information, we enter the value:

$$
\Delta \Gamma=\frac{\Delta E \Delta t}{S_{0}}
$$

Then under the information we will understand the discrete value:

$$
I=\log _{2} \Delta \Gamma
$$

A complex system is a collection of subsystems $(1,2, \ldots, \mathrm{k}, \ldots \mathrm{N})$, so the total amount of information of a complex system will be given by the formula:

$$
I=\sum I_{k}
$$

We note that the size of the unit cell of the exaggeration of the degrees of freedom decreases according to the exponential law $(h<<1)$. Therefore, in macroscopic systems, the discreteness of information associated with the uncertainty of the system state will become insignificant [19].

\subsection{Brownian motion. The law of increasing information}

As it was shown in the previous paragraph, any system from the point of view of a remote macroscopic observer experiences random energy and momentum jumps, which corresponds to the Brownian motion of the particle. This representation allows building a model of increasing information system at the microscopic level. Let us note that the law of information growth associated with the second beginning of thermodynamics is usually considered at the micro level ([8]p.47). The use of the proposed model seems to the authors to be a promising direction for the general proof of the second principle of thermodynamics at the micro level.

We consider a mathematical model of the evolution of a quantum particle in phase space $M^{2 n}(p, q)$. Here $\mathrm{p}$ is the generalized momentum, $\mathrm{q}$ is the generalized coordinate. To describe the evolution, we assume that the particle motion is described by the diffuse Ito process ([9], p. 141). Also we assume that the appropriate selection of the system of units can achieve that $p_{k}=q_{k}$. In this case, we assume that the phase space is divided into concentric balls with volumes $\omega, 2 \omega, \ldots, \mathrm{n} \omega, \ldots$ [4]. In this case, the radius of the balls will be expressed in terms of $\sqrt[n]{\frac{S_{0}}{4 \pi}}$, $\sqrt[n]{\frac{2 S_{0}}{4 \pi}}, \ldots, \sqrt[n]{\frac{S_{0}}{4 \pi}}, \ldots$. Let us note that as the cell number increases, the phase volume and the sphere radius tend to infinity. At the same time, the condition is true:

$$
\lim _{n \rightarrow \infty}\left(R_{k}-R_{k-1}\right)=0
$$

This suggests that if the points of the phase space $A\left(p_{A}, q_{A}\right)$ and $B\left(p_{B}, q_{B}\right)$ are located far enough from each other (the number of elementary cells between them is large enough), the evolution of the system will be characterized by a continuous deterministic process.

Instead of the usual time, we use the notion of the moment of the first exit of the Ito diffuse process from the k-th ball.

Let the particle be at a distance $a$ from the center of the balls.

Definition 1. The first moment of the exit of the Ito process from the ball $R_{k}^{2 n}$ is called a random variable $\tau\left(R_{k}^{2 n}\right)$ such that:

$$
M\left(\tau\left(R_{k}^{2 n}\right)\right)=\frac{1}{2 n}\left(R_{k}^{2}-a^{2}\right)
$$

We determine the probability that a free particle in the process of evolution will fall from point A of the phase space to point $\mathrm{B}$. And $A=R_{m}, B=R_{m-k}$.

Let $f=f_{m, k}$ be a class function $C^{2}$ with compact support, which at $R_{m} \geq|x| \geq R_{m-k}$ is defined by the function

$f(x)=|x|^{2-m}$

From the Dynkin formula, it follows that 


$$
M\left(f\left(B_{\tau\left(R_{m}\right)}\right)=f\left(R_{m}\right)\right.
$$

Let us denote

$$
\begin{aligned}
& p_{k}=p^{B}\left(\left|B_{\tau\left(R_{m}\right)}\right|=R_{m-k}\right), \\
& q_{k}=p^{B}\left(\left|B_{\tau\left(R_{m}\right)}\right|=R_{m}\right) .
\end{aligned}
$$

Taking into account that $p_{k}+q_{k}=1$, we get:

$\lim _{k \rightarrow \infty} p_{k}=\left(\frac{R_{m}}{R_{m-k}}\right)^{2-2 n}$.

Thus, at $\mathrm{n}>2$, for example, when moving on a two-dimensional crystal ([14], [17]), the evolution of the system becomes irrevocable. It is aimed in the direction of increasing R. This increases the phase volume, which increases the entropy of the microsystem. This is in full agreement with the second beginning of thermodynamics, which is usually formulated for macrosystems [4]. The only case when the system is returned is the case $n=1$ [13].

We consider it separately.

$\lim _{k \rightarrow \infty} p_{k}=1$

This result can be interpreted as follows. In one-dimensional systems, the states of the system are distributed in the finite area of the phase space. This in turn means that stationary states are possible in the phase space of such a system [15].

\subsection{Complex system crisis}

Under the complex system crisis, we will understand a fairly rapid restructuring of its external or internal structure. In this case, the system goes from one stable state to another. Such a transition can be influenced by both external and internal factors.

According to the results of the previous paragraphs, such a transition is most likely to be influenced by one factor. If there are a sufficiently large number of factors, the system simply increases its entropy, approaching the equilibrium state; stable configurations are "blurred".

This statement is fully consistent with the so-called principles of synergetics. According to them, the functioning of the system can be described through the control and subordinate modes. The total number of modes can be large, but modes responsible for the crisis (control modes) are a little. Often there is one ([11]p.108).

To move to the macroscopic description of the crisis, we use Langevin equations, which are darkly related to Brownian motion. In this view of the microscopically of the system it can be considered that the model is continuous.

We write the Langevin equations as follows ([11]p.65)

$\dot{\xi}_{n}=\lambda_{n} \xi_{n}+N_{n}\left(\xi_{n}, \xi_{s}\right)+F_{n}(t)$,

$\dot{\xi}_{s}=\lambda_{s} \xi_{s}+N_{s}\left(\xi_{n}, \xi_{s}\right)+F_{s}(t)$.

The value $\xi_{\mathrm{n}}$ we call amplitudes of modes of order, $\xi_{\mathrm{n}}$ amplitudes of subordinate modes, N (q, $\alpha)$ - deterministic (generally nonlinear) function, $\mathrm{F}(\mathrm{t})$ - fluctuating force.

We study in detail the model (*) for one parameter.

$\dot{\xi}=\lambda \xi+N\left(\xi, \xi_{s}\right)+F(t)$

Following the basic provisions of the LGD theory, we consider three main cases ([11]p.110).

1. If $N\left(\xi, \xi_{s}\right)=0$, then $\dot{\xi}=\lambda \xi+F(t)$.

This case corresponds to the case of the usual Brownian motion. Under its influence, the system has one stable state corresponding to the global minimum of the generalized thermodynamic potential $\Phi$ (Fig. 2).

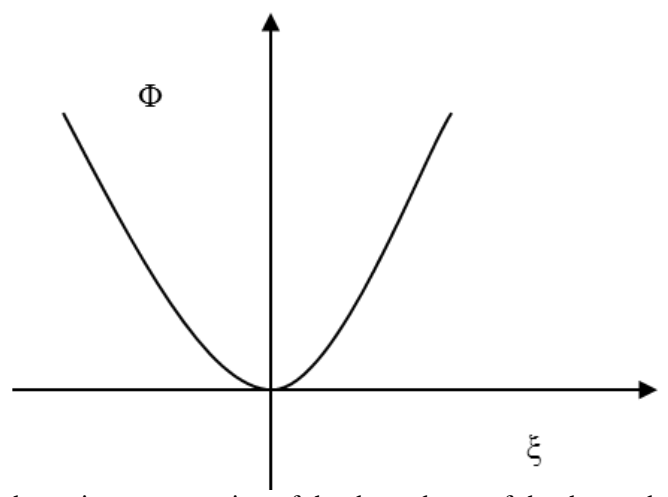

Fig. 2: Schematic representation of the dependence of the thermodynamic potential of the system $\Phi$ on the parameter $\xi$

In this case, the crisis of the system is impossible.

2. If $N\left(\xi, \xi_{s}\right)=\beta \xi^{3}$. Then

$\dot{\xi}=\lambda \xi-\beta \xi^{3}+F(t)$

We assume that the fluctuating force is governed by the Gaussian distribution.

Then the corresponding Fokker-Planck equation has the form:

$f(\xi, t)=\frac{\partial}{\partial \xi}\left[\left(\lambda \xi-\beta \xi^{3}\right) f\right]+\frac{Q}{2} \frac{\partial^{2}}{\partial \xi^{2}} f$.

Here $\mathrm{f}$ - distribution function.

In the stationary case, we obtain

$f=N \exp \left\{Q^{-1}\left(\lambda \xi^{2}-\frac{1}{2} \beta \xi^{4}\right)\right\}$.

Here the value $Q^{-1}\left(\lambda \xi^{2}-1 / 2 \beta \xi^{4}\right)$ plays the role of generalized thermodynamic potential

$\Phi(\xi)=Q^{-1}\left(-\lambda \xi^{2}+\frac{1}{2} \beta \xi^{4}\right)$.

We investigate the form of the function $\Phi(\xi)$ depending on the sign of the parameter $\lambda$.

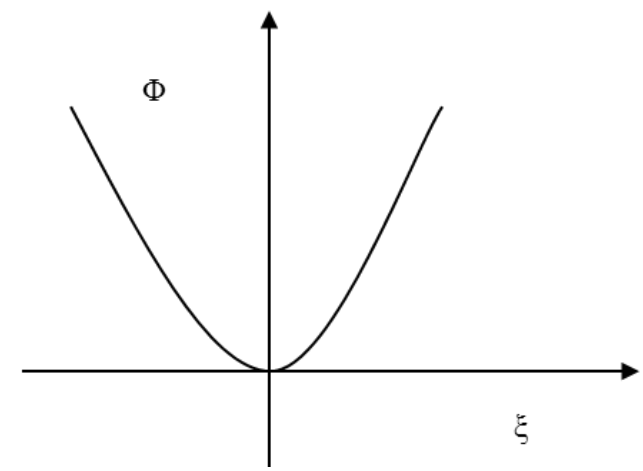

Fig. 3: Schematic representation of the dependence of the thermodynamic potential of the system $\Phi$ on the parameter $\xi$ at $\lambda \leq 0$

If $\lambda \leq 0$. Then there is one global minimum at $\xi=0$ (Fig. 3), which corresponds to the stability of the initial phase A.

If $\lambda>0$. Then the function $\phi(\xi)$ has two global minima at values 


$$
\xi_{1,3}= \pm \sqrt{\frac{\lambda}{\beta}}
$$

and one local maximum $\xi_{2}=0$ (Fig. 4).

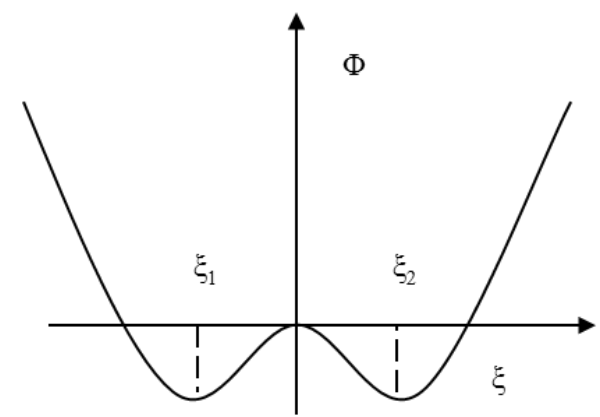

Fig. 4: Schematic representation of the dependence of the thermodynamic potential of the system $\Phi$ on the parameter $\xi$ at $\lambda>0$

This corresponds to the fact that the initial phase A becomes unstable and the system goes into phase $\mathrm{B}$, two realizations (bifurcations) are possible. In this case, the transition is smooth.

1. If

$\dot{\xi}=\lambda \xi-\beta \xi^{3}+\gamma \xi^{5}+F(t)$.

In this case, the Fokker-Planck equation has the form

$f^{\prime}(\xi, t)=\frac{\partial}{\partial \xi}\left[\left(\lambda \xi-\beta \xi^{3}+\gamma \xi^{5}\right) f\right]+\frac{Q}{2} \frac{\partial^{2}}{\partial \xi^{3}} f$

In the stationary case, the thermodynamic potential is given by the formula

$\Phi(\xi)=-Q^{-1}\left(\lambda \xi^{2}-\frac{\beta}{2} \xi^{4}+\frac{\gamma}{3} \xi^{6}\right)$

The dependence of the function $\Phi(\xi)$ depending on the value of the control parameter $\lambda$ is shown in Fig. $5 a, 5 b, 5 c$.

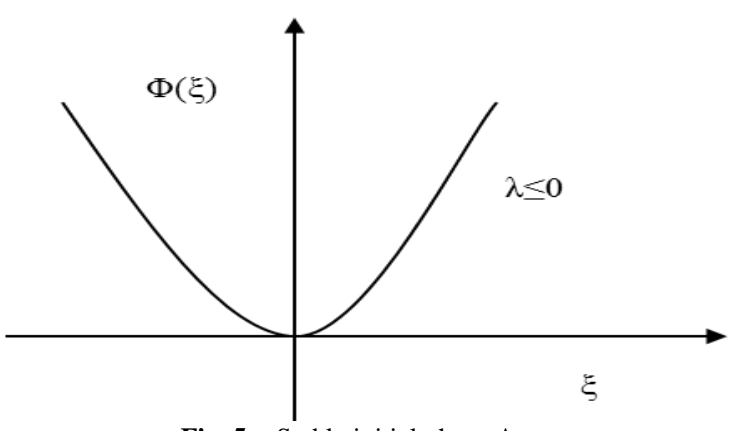

Fig. 5a: Stable initial phase A

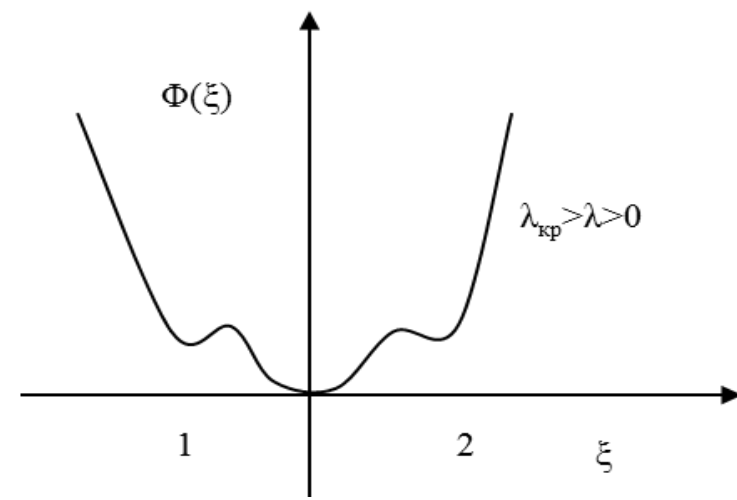

Fig. 5b: Energetically favorable phase A. Local minima 1 and 2 correspond to the metastable state of the phase B

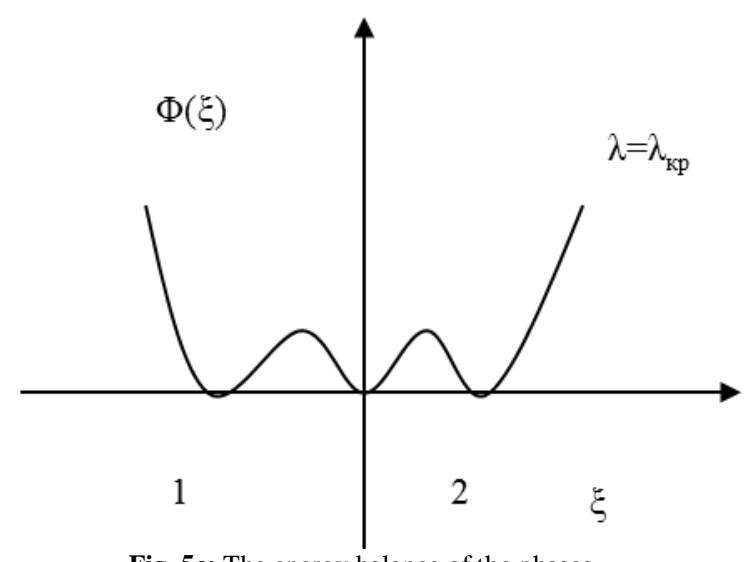

Fig. 5c: The energy balance of the phases

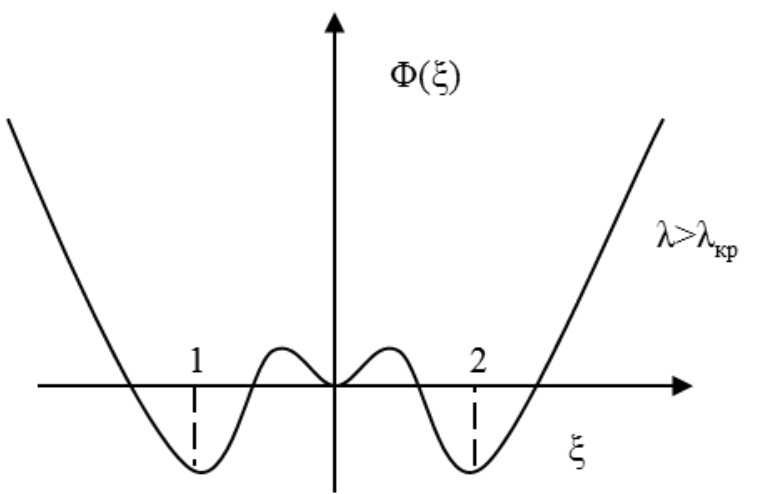

Fig. 5: Phase B (global minima 1 and 2 ) is energetically advantageous. The local minimum 0 corresponds to the metastable state of phase A

Thus, in such a crisis, the change of phases occurs with some delay, which generates the phenomenon of hysteresis, which is a characteristic feature of the transition of the $1^{\text {st }}$ kind.

\subsection{Mesodynamics of crisis}

The studies allowed reducing the main types of crises of complex systems to the well-known theory of LGD, used to describe the phase transitions.

As for the dynamics of the crisis, we can draw the following conclusions ([1], [2]).

1. The crisis of the type (9) is characterized by a smooth flow, which allows it to be controlled throughout its entire length ([1] p.73)(Fig. 6).

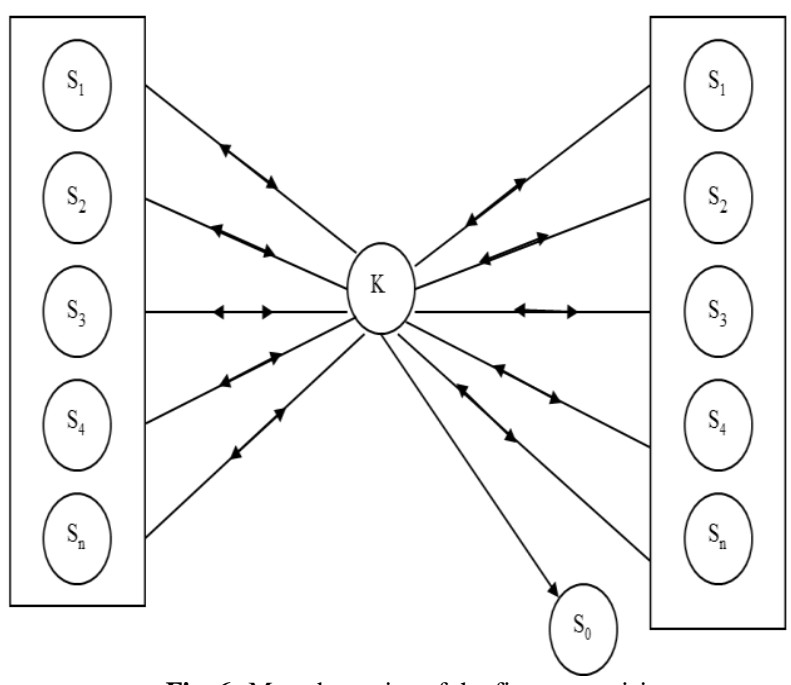

Fig. 6: Mesodynamics of the first type crisis 
Here $\mathrm{S}_{\mathrm{n}}$ - system macro states, $\mathrm{S}_{0}$-system death

2. The crisis is characterized by "overheating" of the system. As a result, the crisis becomes irreversible, poorly managed, and passes quickly enough (Fig. 7).

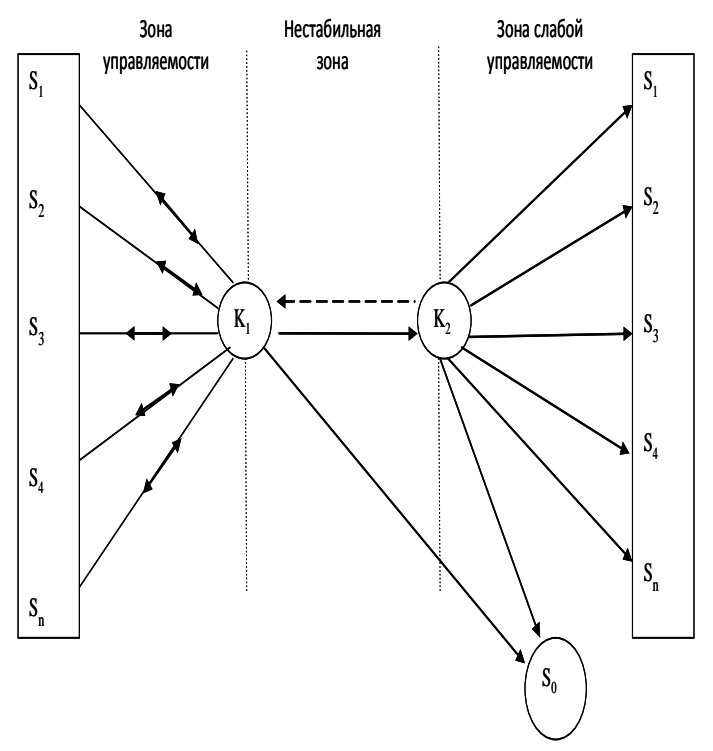

Fig. 7: Mesodynamics of the second type crisis

\section{Conclusions}

The work offers a theoretical basis for the development of mathematical models and control methods for complex systems of different nature. It deals with the functioning of the system in a crisis from the point of remote observer. The micro-level of the system is considered, the transition to the macro-level and to the aerodynamics of the process is carried out. At the same time, the authors tried to use a simple, convenient and, at the same time, adequate mathematical apparatus.

In conclusion, we give some recommendations on the use of the results.

It should be noted that in the first part the usual notion of time was not used and the space of generalized coordinates was not considered separately. This approach will be useful when working on quantization of fields of different nature, as it eliminates the appearance of singularity.

The conclusion about the "dominance" of Brownian motion in any real system shows that in order to transfer a complex social system to a new stable position, external control must work with sufficiently large objects of the system. However, these objects should not be comparable to the system itself. In this case, a managed crisis would be too energy-consuming. This position justifies the theory of cluster management ([5], [6]).

In particular, the cluster approach is currently being implemented to restore flax growing in Smolensk region.

\section{References}

[1] Adigamov A.E., Belokopytov A.V., Yudenkov A.V. Modeling of anti-crisis management on the basis of system analysis // Mining analytical bulletin (scientific and technical journal), 2009. No. 7 . P.42-47.

[2] Adigamov A.E., Sapkina E.A., Yudenkov A.V. Mathematical modeling of conflict situations between the subjects of the agricultural market on the example of processing enterprises of Smolensk region. // Mining information-analytical bulletin (scientific-technical journal), 2012. No. 6. P. 363-365.
[3] Arzhakova N.V., Novoseltsev V.I., Redkozubov S.A. Market dynamics management: system approach - Voronezh: Publ. house of Voronezh State University, 2004. - 192 p.

[4] Volodchenkov A.M., Yudenkov A.V., Rimskaya L.P. Quantization of information in the symplectic variety // In the collection: Socioeconomic development of the region: experience, problems, innovations (materials of the VI International scientific-practical conference in the framework of Plekhanov's spring and the 110th anniversary of the University). Ministry of education and science of the Russian Federation. G.V. Plekhanov Russian University. Smolensk branch, 2017. P. 41-46.

[5] Kovaleva A.E. Theoretical aspects of cluster management in agroindustrial complex. // Economics and entrepreneurship, 2016. № 12 (66-2). P. 41-43.

[6] Kovaleva A.E. Cluster management of the agricultural sector in terms of investment instability // Science and business: ways of development, 2016. No. 2. P. 19-22.

[7] Landau L.D., Lifshitz E.M. Theoretical mechanics Vol. 3. Quantum mechanics - M.: Nauka, 1989. -768 p.

[8] Landau L.D., Lifshitz E.M. Theoretical mechanics Vol. 5. Statistical mechanics - M.: Nauka, 1989. -626 p.

[9] Oksendal B. Stochastic differential equations. - M: Mir, Publishing house AST, 2003. $-408 \mathrm{p}$.

[10] Pugachev V.S., Sinitsyn I.N. The theory of stochastic systems. Textbook.- Moscow: Logos, 2004. - 1000p..

[11] Haken G. Information and self-organization. - M: KomKniga, 2005. $-248 \mathrm{p}$.

[12] Yudenkov A.V. Brownian motion of microparticles on a discrete symplectic phase space // In the book: Food safety: from dependence to independence. Proceedings of the international scientific-practical conference, 2017. P. 712-714.

[13] Port S., Stone C. (1979): Brownian Motion and Classical Potential Theory. Academic Press.

[14] 14. Geim A.K. Graphene prehistory // Physica Scripta. 2012. № T146. C. 140-143.

[15] 15. Shirokov B.M., Gromakovskaya L.A. Distribution of values of the sum of unitary divisors in residue classes // Проблемы анализа, 2016. T. 5 (23). № 1. C. 31-44.

[16] 16. Logachev O.A. On the local invertibility of finite automata with no loss of information // Applied discrete mathematics, 2018. No. 39. P. 78-93.

[17] 17. Kochnev A.S., Ovid'ko I.A., Semenov B.N., Sevastyanov Ya.A. Mechanical properties of graphene containing elongated tetravacancies (575757-666-5757 defects) // Reviews on Advanced Materials Science, 2017. T. 48. № 2. C. 142-146.

[18] 18. Bure V.M., Yefimov A.N., Karelin V.V. Stationary cycles in the deterministic service system // Vestnik of St. Petersburg University. Applied mathematics. Informatics. Management processes, 2018. Vol. 14. No. 1. P. 40-50.

[19] 19. Karelin V.V., Bure V.M., Svirkin M.V. Generalized model of information dissemination in continuous time // Bulletin of St. Petersburg University. Applied mathematics. Informatics. Management processes, 2017. Vol.13. No. 1. P. 74-80.

[20] 20. Belyaeva M.V., Mitrofanov M.Yu. New results in the theory of search // Discrete analysis and operations research. Series 2, 2004. Vol. 11. No. 1. P. 26-50.

[21] 21. Bartlett J.G., Bucher M., Cardoso J.-F., Castex G., Delabrouille J., Ganga K., Gauthier C., Giraud-Héraud Y., Karakci A., Le Jeune M., Patanchon G., Piat M., Remazeilles M., Roman M., Rosset C., Roudier G., Stompor R., Lähteenmäki A., León-Tavares J., Tornikoski M. et al. Planck 2015 results: I.overview of products and scientific results // Astronomy and Astrophysics, 2016. T. 594. C. A1. 